\title{
DISTANCE-BASED SHAPE STATISTICS
}

Guillaume Charpiat

\author{
Équipe Odyssée \\ École Normale Supérieure \\ 45 rue d'Ulm \\ 75005 Paris, France \\ charpiat@di.ens.fr \\ pmaurel@di.ens.fr
}

Olivier Faugeras

Renaud Keriven

Pierre Maurel

\author{
Équipe Odyssée \\ INRIA Sophia-Antipolis \\ 2004 route des Lucioles, BP 93 \\ 06902 Sophia-Antipolis Cedex, France \\ faugeras@sophia.inria.fr
}

\author{
Équipe Odyssée \\ ENPC \\ 6 av Blaise Pascal \\ 77455 Marne la Valle, France \\ keriven@certis.enpc.fr
}

\begin{abstract}
This article deals with statistics on sets of shapes. The approach is based on the Hausdorff distance between shapes. The choice of the Hausdorff distance between shapes is itself not fundamental since the same framework could be applied with another distance. We first define a smooth approximation of the Hausdorff distance and build non-supervised warpings between shapes by a gradient descent of the approximation. Local minima can be avoided by changing the scalar product in the tangent space of the shape being warped.When non-supervised warping fails, we present a way to guide the evolution with a small number of landmarks. Thanks to the warping fields, we can define the mean of a set of shapes and express statistics on them. Finally, we come back to the initial distance between shapes and use it to represent a set of shapes by a graph, which with the technic of graph Laplacian leads to a way of projecting shapes onto a low dimensional space.
\end{abstract}

\section{SHAPES AND SHAPE METRICS}

Fully defining the notion of shape is beyond the scope of this article in which we use a limited, i.e purely geometric, definition. In our context we define a shape $\Gamma$ to be the boundary of a regular and bounded subset of $\mathbb{R}^{n}$. Since we are driven by image applications we also assume that all our shapes are contained in a hold-all regular open bounded subset of $\mathbb{R}^{n}$ which we denote by $\Omega$. We suppose $\Gamma$ to be a smooth codimensionone manifold of $\mathbb{R}^{n}$, and denote by $S$ the set of shapes. We refer the reader to [1] for a more rigorous and complete analysis.

Since we want to be able to compare shapes, a way to quantify the similarity between them must be defined. One of the broadly used distance between shapes is the Hausdorff distance:

$$
d_{H}\left(\Gamma_{1}, \Gamma_{2}\right)=\max \left\{\sup _{\mathbf{x} \in \Gamma_{1}} d_{\Gamma_{2}}(\mathbf{x}), \sup _{\mathbf{x} \in \Gamma_{2}} d_{\Gamma_{1}}(\mathbf{x})\right\}
$$

where $d_{\Gamma}$ is the distance function to the shape $\Gamma$ :

$$
d_{\Gamma}(\mathbf{x})=\inf _{\mathbf{y} \in \Gamma} d(\mathbf{x}, \mathbf{y}) .
$$

Some other distances are based on signed distance functions. The signed distance function to a shape $\Gamma$, denoted by $\tilde{d}_{\Gamma}$, is equal to $d_{\Gamma}$ outside $\Gamma$ and equal to $-d_{\Gamma}$ inside $\Gamma$. A possible shape distance is then the norm of the Sobolev space, $W^{1,2}(\Omega)$, of square integrable functions with square integrable derivatives:

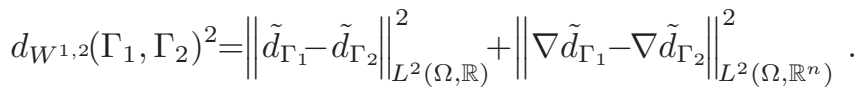

\section{VARIATIONAL SHAPE WARPING}

We assume that we are given a function $E: S \times S \rightarrow \mathbb{R}^{+}$, the energy. This energy can be thought of as a measure of dissimilarity between two shapes. Warping a shape $\Gamma_{1}$ into another one $\Gamma_{2}$ can be stated as the minimization of the energy $E\left(., \Gamma_{2}\right)$ starting from $\Gamma_{1}$, i.e., finding a family of shapes $\{\Gamma(t), t \geq 0\}$ with $\Gamma(0)=\Gamma_{1}$ and $\Gamma(t)$ following some gradient descent toward $\Gamma_{2}$.

\subsection{Shape gradient}

In order to define the gradient of the energy functional, the first step is to compute its Gâteaux derivatives in all directions, i.e., for all admissible velocity fields $\mathbf{v}: \Gamma \rightarrow \mathbb{R}^{n}$. Let us denote by $\mathcal{G}_{\Gamma}\left(E\left(\Gamma, \Gamma_{2}\right), \mathbf{v}\right)$ the Gâteaux derivatives of the energy function $E\left(\Gamma, \Gamma_{2}\right)$ with respect to the shape $\Gamma$ and in the direction $\mathbf{v}$ :

$$
\mathcal{G}_{\Gamma}\left(E\left(\Gamma, \Gamma_{2}\right), \mathbf{v}\right)=\lim _{\varepsilon \rightarrow 0} \frac{E\left(\Gamma+\varepsilon \mathbf{v}, \Gamma_{2}\right)-E\left(\Gamma, \Gamma_{2}\right)}{\varepsilon} .
$$

We would like to pick the gradient as the direction of steepest descent of the energy. But, to be able to assess the steepness of the energy, the deformation space needs to be equipped with an inner product. We model the space of admissible deformations as an inner product space $\left(F,\langle,\rangle_{F}\right)$. Under certain regularity conditions, there exists a vector $\mathbf{w} \in F$ such that:

$$
\forall \mathbf{v} \in F, \mathcal{G}_{\Gamma}\left(E\left(\Gamma, \Gamma_{2}\right), \mathbf{v}\right)=\langle\mathbf{w} \mid \mathbf{v}\rangle_{F} .
$$


We call it the shape gradient of E relative to the inner product $\langle,\rangle_{F}$ and we note it $\mathbf{w}=\mathbf{D}_{\Gamma}^{\left(F,\langle,\rangle_{F}\right)} E\left(\Gamma, \Gamma_{2}\right)$. Usually $F$ is taken as the set $L^{2}\left(\Gamma, \mathbb{R}^{n}\right)$ of the square integrable velocity fields on $\Gamma$, and $\langle,\rangle_{F}$ its associated inner product:

$$
\langle f \mid g\rangle_{L^{2}}=\int_{\Gamma} f(\mathbf{x}) \cdot g(\mathbf{x}) d \Gamma(\mathbf{x}) .
$$

In that case, we will only denote the gradient by $\mathbf{D}_{\Gamma} E\left(\Gamma, \Gamma_{2}\right)$.

Equipped with some shape gradient, we can define the warping of a shape $\Gamma_{1}$ into another one $\Gamma_{2}$ as finding the family $\Gamma(t)$ solution of the following Partial Differential Equation:

$$
\begin{aligned}
& \Gamma(0)=\Gamma_{1} \\
& \frac{\partial \Gamma}{\partial t}=-\mathbf{D}_{\Gamma}^{\left(F,\langle,\rangle_{F}\right)} E\left(\Gamma, \Gamma_{2}\right) .
\end{aligned}
$$

Natural candidates for the energy function $E$ are the distances presented in the previous section. The Hausdorff distance is not Gâteaux differentiable. Yet, this problem can be solved using smooth approximation of this distance, denoted by $\tilde{d}_{H}\left(\Gamma_{1}, \Gamma_{2}\right)$, which presents the advantage of being differentiable. This approximation is obtained using the following property (see [2] for more details): for any $f$ integrable on the compact set $\Gamma$, we have

$$
\lim _{\beta \rightarrow+\infty}\left(\frac{1}{|\Gamma|} \int_{\Gamma} f(x)^{\beta} d \Gamma(x)\right)^{1 / \beta}=\sup _{x \in \Gamma} f(x) .
$$

A few steps of the result of warping a silhouette of a fish onto another one, using the approximation of the Hausdorff distance as the energy function, are shown in Fig. 1.

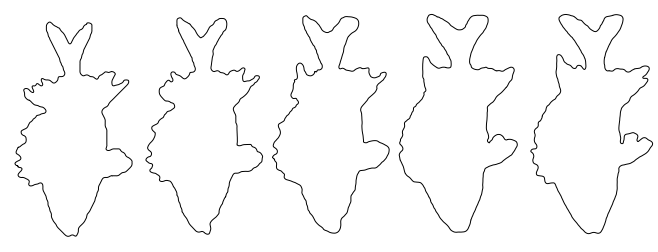

Fig. 1. Hausdorff warping of a fish onto another.

\subsection{Generalized gradient and spatially coherent flows}

Although mathematically well justified, the warpings induced by $E=\tilde{d}_{H}$ or $E=d_{W^{1,2}}$ are not always completely satisfying: the obtained deformations do not seem to be the one a human observer would have chosen. To cope with this, a way to favor rigid (translations and rotations) and scaling motions is introduced in [3]. This approach consists in changing the inner product used in the definition of the gradient. We decompose the deformation space $L^{2}$ into a sum of several mutually orthogonal linear subspaces: the subspace $T$ of translations, the subspace $R$ of rotations around the centroid, the subspace $S$ of scaling motions centered on the centroid and the orthogonal complement of these subspaces $G$ :

$$
L^{2}=T \oplus R \oplus S \oplus G
$$

Applying different penalty factors to the different types of motions, we define a new inner product related to this decomposition and these penalty factors. The gradient of the Energy function relative to this new inner product can be easily deduced from the standard $L^{2}$-gradient $\mathbf{D}_{\Gamma} E$ (see [3] for more details). The resulting warping promotes spatially coherent motions, keeping the warping of small details for the end of the evolution. In Fig. 2, we show a three-dimensional warping example from a teddy bear to Hayao Miyazaki's character Totoro. We use here the $d_{W^{1,2}}$ distance defined in Section 1. The $L^{2}$ gradient descent is unable to give satisfying results. The modified gradient descent favoring rigid plus scaling motions leads to better results.
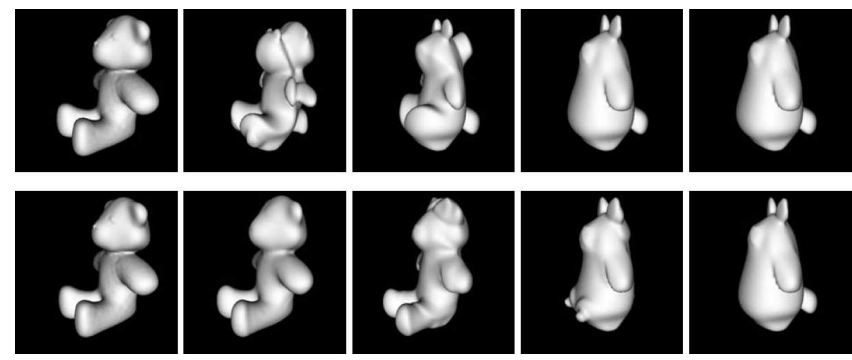

Fig. 2. 3D shape warping with the $L^{2}$ gradient descent (top) and with a modified gradient descent favoring rigid plus scaling motions.

Actually, only global coherent motions are promoted by this new gradient. The symptom of "unnatural" warping persists in the worst cases, such as complex shapes or shapes related by an articulated motion.

\section{LANDMARKS}

Landmarks are then necessary in worst cases. Provided by the user (anatomical landmarks), or automatically extracted (geometric landmarks), we assume that we are given $p$ pairs of corresponding points on the initial and on the target shapes, $\left\{\left(\mathbf{x}_{1 i}, \mathbf{x}_{2 i}\right) \in \Gamma_{1} \times \Gamma_{2}, 1 \leq i \leq p\right\}$. We would like to use the information given by these correspondences to guide the evolution. We do this by adding a landmark term to the energy:

$$
E_{t o t}\left(\Gamma(t), \Gamma_{2}\right)=E\left(\Gamma(t), \Gamma_{2}\right)+\sum_{i=1}^{p} d\left(\mathbf{x}_{i}(t), \mathbf{x}_{2 i}\right)^{2} .
$$

where $x_{i}(t)$ is the $i$ th landmark on $\Gamma(t)$. In particular, we have $x_{i}(0)=x_{1 i}$.

Formally, this energy yields Dirac peaks in the expression of the gradient of the energy. This is indeed not a good candidate for a gradient descent. The solution is again to change the inner product which appears in the definition of the gradient. Let $H^{1}\left(\Gamma, \mathbb{R}^{n}\right)$ be the Sobolev space of square integrable velocity fields with square integrable derivatives. We consider the canonical inner product of $H^{1}\left(\Gamma, \mathbb{R}^{n}\right)$ :

$\langle f \mid g\rangle_{H^{1}}=\int_{\Gamma} f(\mathbf{x}) \cdot g(\mathbf{x}) d \Gamma(\mathbf{x})+\int_{\Gamma} \nabla_{\Gamma} f(\mathbf{x}) \cdot \nabla_{\Gamma} g(\mathbf{x}) d \Gamma(\mathbf{x})$

where $\nabla_{\Gamma} f$ and $\nabla_{\Gamma} g$ are respectively the intrinsic derivatives on $\Gamma$. 
Interestingly, the $H^{1}$ gradient can be obtained from the $L^{2}$ gradient by solving an intrinsic heat equation with a data attachment term (see [4] for more details): $\mathbf{D}_{\Gamma}^{H^{1}} E_{t o t}$ is solution of

$$
\Delta_{\Gamma} u=u-\mathbf{D}_{\Gamma}^{L^{2}} E_{t o t}
$$

where $\Delta_{\Gamma}$ denotes the intrinsic Laplacian operator on the surface, often called the Laplace-Beltrami operator. The solution of this equation coincides with that of the following variational problem:

$\underset{u}{\arg \min } \int_{\Gamma}\left|u(\mathbf{x})-\mathbf{D}_{\Gamma}^{L^{2}} E_{t o t}(\mathbf{x})\right|^{2} d \Gamma(\mathbf{x})+\int_{\Gamma}\left|\nabla_{\Gamma} u(\mathbf{x})\right|^{2} d \Gamma(\mathbf{x})$

and the $H^{1}$ gradient is finally a smoothed version of the $L^{2}$ gradient. Fig. 3 shows the warping between two hand shapes. The energy $E=d_{W^{1,2}}$ yields an unnatural warping. With three landmarks and spatially coherent flows, a satisfying warping is recovered.
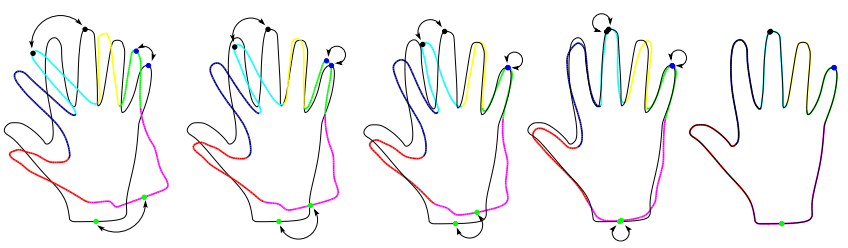

Fig. 3. evolution with $E=d_{W^{1,2}}$ plus coherent flows plus three provided landmarks.

\section{MEAN, COVARIANCE AND MODES OF VARIATION}

We have now developed the tools for defining several concepts relevant to a theory of stochastic shapes as well as providing the means for their effective computation. The first obvious concept is that of the mean of a set of shapes.

Definition 1 Given $\Gamma_{1}, \cdots, \Gamma_{N}, N$ shapes, we define their mean as any shape $\hat{\Gamma}$ that minimizes the function $\mu: S \rightarrow \mathbb{R}^{+}$ defined by $\mu\left(\Gamma, \Gamma_{1}, \cdots, \Gamma_{N}\right)=\frac{1}{N} \sum_{i=1, \cdots, N} E\left(\Gamma, \Gamma_{i}\right)$

An algorithm for computing approximations to a mean readily follows from the previous section: start from an initial shape and solve the PDE: $\Gamma_{t}=-\nabla \mu\left(\Gamma, \Gamma_{1}, \cdots, \Gamma_{N}\right) \mathbf{n}$. We show some examples in Fig. 4.

We can now define the covariance of $N$ shapes and their modes of variation (see [2] for justifications).

Definition 2 Given $N$ shapes $\Gamma_{i}$, we note $\beta_{i}$ the normal velocity fields of the gradients of the functions $\Gamma \rightarrow \tilde{d}_{H}\left(\Gamma, \Gamma_{i}\right)$ and $\hat{\beta}=\frac{1}{N} \sum_{i=1}^{N} \beta_{i}$ their mean. The covariance operator of these $N$ shapes for their mean $\hat{\Gamma}$ is the linear continuous operator of $L^{2}(\hat{\Gamma})$ defined by:

$$
\Lambda(\beta)=\sum_{i=1, N}<\beta, \beta_{i}-\hat{\beta}>_{\hat{\Gamma}}\left(\beta_{i}-\hat{\beta}\right) .
$$

Definition 3 Let $\hat{\Lambda}$ be the $N \times N$ symmetric semi positive definite matrix $\hat{\Lambda}$ defined by $\hat{\Lambda}_{i j}=<\beta_{i}-\hat{\beta}, \beta_{j}-\hat{\beta}>_{\hat{\Gamma}}$. Let $p \leq N$ be its rank, $\sigma_{1}^{2} \geq \sigma_{2}^{2} \geq \cdots \geq \sigma_{p}^{2}>0$ its positive eigenvalues and $\mathbf{u}_{1}, \cdots, \mathbf{u}_{N}$ the corresponding eigenvectors. Let $u_{i j}$ be the ith coordinate of the vector $\mathbf{u}_{j}$ and $v_{j}$ be defined by $v_{j}=\frac{1}{\sigma_{j}} \sum_{i=1}^{N} u_{i j}\left(\beta_{i}-\hat{\beta}\right)$. The velocities $v_{k}, k=1, \cdots, p$ can be interpreted as modes of variation of the shapes and the $\sigma_{k}^{2}$ 's as variances for these modes. Looking at how the shape varies for the kth mode is equivalent to solving the PDEs $\Gamma_{t}= \pm v_{k}(\Gamma) \mathbf{n}$ with $\Gamma(0,)=.\hat{\Gamma}($.$) .$

Examples of theses modes for the cases of the fingers and of the ten fishes is shown in Fig. 5.
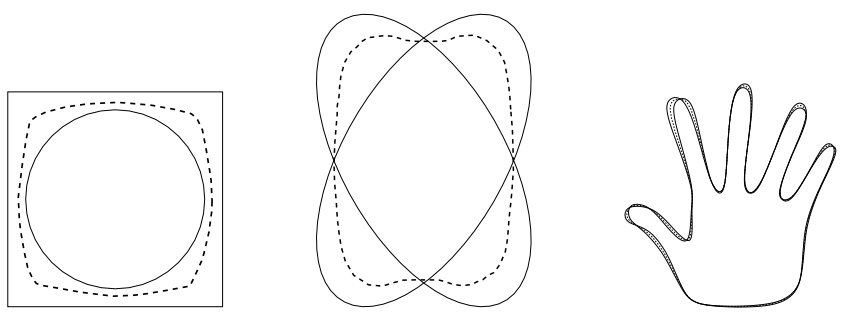

Fig. 4. Examples of means of several curves: a square and a circle (left), two ellipses (middle) and two hands (right).

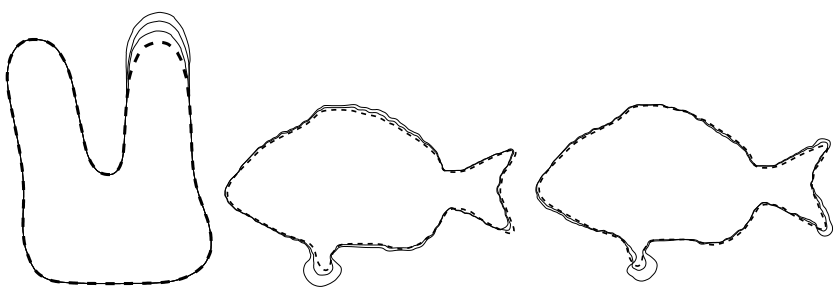

Fig. 5. The first mode of variation in the finger case and the first two ones for ten sample shapes of fish.

\section{GRAPH LAPLACIAN}

We can interpret the previous section as a method to associate to any shape from a given set some coordinates in a low-dimensional linear space. Indeed, for any choice of dimension $m$, we can keep from a set of shapes only its $m$ first eigenmodes of deformation $\left(e_{1 \leqslant i \leqslant m}\right)$ (the most significant ones, i.e., the ones with highest eigenvalues), which form an orthogonal family in the tangent space of the mean shape $\hat{\Gamma}$ embedded with its inner product. Then to a shape $\Gamma$ we associate the warping field $e_{\Gamma}=\nabla_{\hat{\Gamma}} d^{2}(\hat{\Gamma}, \Gamma)$ defined on $\hat{\Gamma}$ and to this warping field we associate the coordinates $\left\langle e_{\Gamma} \mid e_{i}\right\rangle$ of its projection onto the family $\left(e_{i}\right)$, i.e., we have associated to any shape $\Gamma$ the coordinates of its projection in a low-dimensional linear space.

In fact, we do not need the computation of any warping field to be able to build a low-dimensional map of a set of 
shapes: even if these fields must carry much information, it is possible to build such a map when given only the distances between shapes, from a purely static geometrical point of view, thanks to the graph Laplacian technic [5].

Let us denote by $\left(\Gamma_{1 \leqslant i \leqslant n}\right)$ a set of $n$ shapes and consider a distance $d$ on this set, for example the Hausdorff distance between shapes. We fix a positive integer $K$ and search for the $K$ nearest neighbors $N_{1 \leqslant l \leqslant K}^{i}$ of each shape $\Gamma_{i}$ for the chosen distance $d$. We then define a symmetric weight matrix $W$ by

$$
W_{i, j}=\delta_{i, j} e^{-\frac{d\left(\Gamma_{i}, \Gamma_{j}\right)^{2}}{2 \sigma^{2}}}
$$

where

$$
\delta_{i, j}= \begin{cases}1 & \text { if } i \in N^{j} \\ 0 & \text { otherwise }\end{cases}
$$

and we have chosen for $\sigma$ the mean distance between neighbors:

$$
\sigma=\frac{\sum_{i, j} d\left(\Gamma_{i}, \Gamma_{j}\right) \delta_{i, j}}{\sum_{i, j} \delta_{i, j}}
$$

Then, let us consider the symmetric negative semi-definite matrix $L=W-D$ where $D_{i, j}=\sum_{i} W_{i, j} \delta_{i, j}:$ it is a discrete approximation of the Laplacian operator. Thus, as explained in [5], its eigenvectors $F_{k}$ of highest (negative) non-zero eigenvalues are the best functions from the shapes $\Gamma_{i}$ to $\mathbb{R}$ that could be used as coordinate system of the set of shapes. Consequently, we obtain a natural map in $\mathbb{R}^{m}$ where each shape $\Gamma_{i}$ is represented by a dot with coordinates $\left(F_{1 \leqslant k \leqslant m}\left(\Gamma_{i}\right)\right)$. Let us try this approach on an artificial dataset. We build a set of rectangles with same center and width but different lengths and orientations, so there are two natural parameters we would expect the algorithm to find. Rectangles are randomly chosen such that the distribution of their corners is the uniform law in the authorized area (orientation between $-\frac{\pi}{6}$ and $+\frac{\pi}{6}$, and length between 2 and 4 times the width). Results vary depending on the distribution density and the value of $K$ : the higher the density, the better the results. Fig. 6 has been computed for 700 rectangles.

Let us now study the more complicated case of some different classes in a same connected component. We consider a set of 111 fish from the same database as before. The resulting map for the two first coordinates (see Fig. 7) shows some clusters of fish families.

\section{CONCLUSION}

We have presented several developments of our shape work, a generalization of our shape gradient, the extension of our framework that includes landmarks, and the application of a technique for dimensionality reduction that seems to offer interesting avenues for future work.

\section{REFERENCES}

[1] M.C. Delfour and J.-P. Zolésio, "Shape analysis via distance functions: Local theory," in Boundaries, interfaces

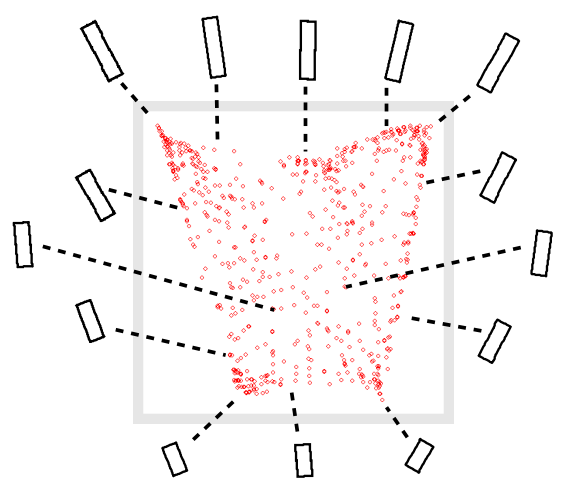

Fig. 6. Map from the graph Laplacian method for a set of rectangles whose length and orientation have been chosen randomly $(K=15)$.

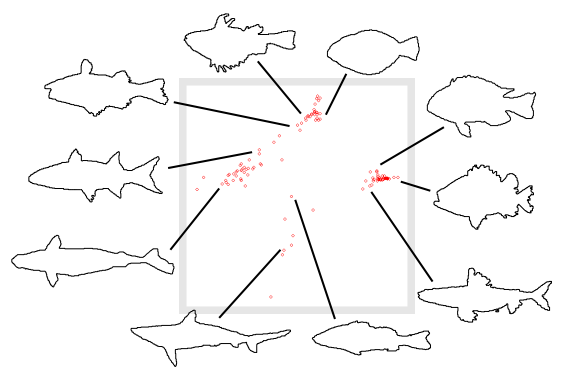

Fig. 7. Two first coordinates for a set of 111 fish from different classes. The elements from each family are got together into clusters $(K=25)$.

and transitions, vol. 13 of CRM Proc. Lecture Notes, pp. 91-123. AMS, Providence, RI, 1998.

[2] G. Charpiat, O. Faugeras, and R. Keriven, "Approximations of shape metrics and application to shape warping and empirical shape statistics," Foundations of Computational Mathematics, vol. 5, no. 1, pp. 1-58, Feb. 2005.

[3] G. Charpiat, R. Keriven, J.P. Pons, and O. Faugeras, "Designing spatially coherent minimizing flows for variational problems based on active contours," in 10th International Conference on Computer Vision, Beijing, China, 2005.

[4] P. Maurel, O. Faugeras, and R. Keriven, "Reconciling landmarks and level sets: geometric shape warping and matching using generalized gradients and correspondence-augmented implicit representations," Tech. Rep. 5726, INRIA, Oct. 2005.

[5] M. Belkin and P. Niyogi, "Laplacian eigenmaps for dimensionality reduction and data representation," 2001. 